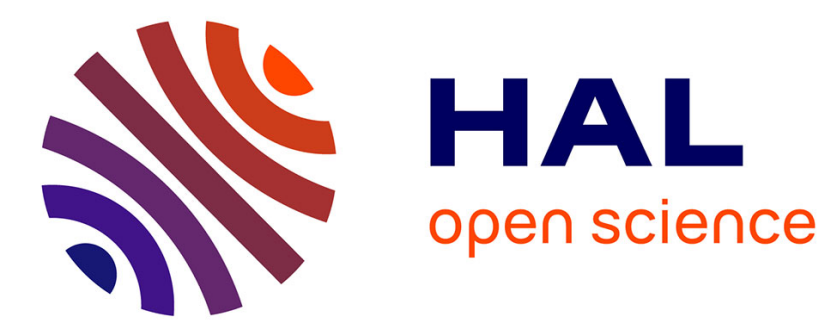

\title{
La place des réformistes dans le mouvement national algérien
}

Malika Rahal

\section{To cite this version:}

Malika Rahal. La place des réformistes dans le mouvement national algérien. Vingtième siècle. Revue d'histoire, 2004, 10.3917/ving.083.0161 . hal-01316739

\section{HAL Id: hal-01316739 \\ https://hal.science/hal-01316739}

Submitted on 17 May 2016

HAL is a multi-disciplinary open access archive for the deposit and dissemination of scientific research documents, whether they are published or not. The documents may come from teaching and research institutions in France or abroad, or from public or private research centers.
L'archive ouverte pluridisciplinaire HAL, est destinée au dépôt et à la diffusion de documents scientifiques de niveau recherche, publiés ou non, émanant des établissements d'enseignement et de recherche français ou étrangers, des laboratoires publics ou privés. 


\title{
LA PLACE DES RÉFORMISTES DANS LE MOUVEMENT NATIONAL ALGÉRIEN
}

\author{
Malika Rahal
}

Presses de Sciences Po (P.F.N.S.P.) | Vingtième Siècle. Revue d'histoire

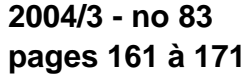

ISSN 0294-1759

Article disponible en ligne à l'adresse:

http://www.cairn.info/revue-vingtieme-siecle-revue-d-histoire-2004-3-page-161.htm

Pour citer cet article :

Rahal Malika, « La place des réformistes dans le mouvement national algérien »,

Vingtième Siècle. Revue d'histoire, 2004/3 no 83, p. 161-171. DOI : 10.3917/ving.083.0161

Distribution électronique Cairn.info pour Presses de Sciences Po (P.F.N.S.P.).

(C) Presses de Sciences Po (P.F.N.S.P.). Tous droits réservés pour tous pays.

La reproduction ou représentation de cet article, notamment par photocopie, n'est autorisée que dans les limites des conditions générales d'utilisation du site ou, le cas échéant, des conditions générales de la licence souscrite par votre établissement. Toute autre reproduction ou représentation, en tout ou partie, sous quelque forme et de quelque manière que ce soit, est interdite sauf accord préalable et écrit de l'éditeur, en dehors des cas prévus par la législation en vigueur en France. II est précisé que son stockage dans une base de données est également interdit. 


\title{
LA PLACE DES RÉFORMISTES DANS LE MOUVEMENT NATIONAL ALGÉRIEN
}

\author{
Malika Rahal
}

À travers l'itinéraire de 'Ali Boumendjel, Malika Rahal propose de revisiter la place du réformisme dans les idées et les pratiques du mouvement national. En revenant sur la fusion de tous les courants dans le FLN, cet article pointe les autres voies qui étaient alors proposées à l'avenir de l'Algérie et que l'histoire officielle a passées sous silence.

\section{O HISTOIRE OFFICIELLE ET UDMA}

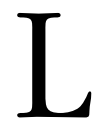

es luttes de libération se sont accompagnées d'un travail d'écriture de l'histoire qui a contribué à la construction de la nation. L'Algérie a connu après l'indépendance un régime de parti unique : l'histoire nationale proposée par le Front de libération nationale s'est imposée comme une histoire officielle proposant une version monolithique et hégémonique de l'histoire du mouvement national et de la guerre de libération.

Cette version de l'histoire est définie précisément dans des textes tels que la Charte d'Alger qui donne, en 1964, des orientations pour le travail des chercheurs. Elle apparaît par la suite dans les manuels scolaires, dans les lieux de mémoire ou les noms des rues. Cette histoire officielle s'articule autour de plusieurs mythes : la glorification de la lutte armée; la référence constante au peuple sous la forme d'un populisme qui sert de principe de base au FLN devenu parti unique au lendemain de l'indépendance ; la définition de la culture algérienne par la langue arabe et la reli- gion musulmane à l'exclusion de toute autre langue ou religion. Par ailleurs, les principes fondant cette mythologie auraient été défendus par un courant politique unique allant de l'Étoile Nord-Africaine au Front de libération Nationale. Ils demeurent fondamentaux dans l'État algérien issu de la guerre de libération ${ }^{1}$.

Comme ailleurs, l'histoire officielle est, en Algérie, une histoire écrite par les vainqueurs, c'est-à-dire par le parti au pouvoir depuis l'indépendance. Il n'y a donc aucune surprise à ce que le FLN produise une histoire qui valorise sa propre position durant le conflit, au détriment des forces politiques concurrentes comme le MNA, le Mouvement national algérien de Messali Hadj. Mais le FLN ne cherche par seulement, à travers l'histoire officielle, à masquer toute concurrence durant le conflit. Il veut également s'enraciner dans un combat politique ancien et unique. Il s'agit pour cela d'occulter la multiplicité des héritages et des traditions politiques développées en Algérie pour revendiquer une filiation linéaire: celle de l'ENA, créée à Paris en 1926, puis du PPA, le Parti populaire algérien, fondé après l'interdiction de l'ENA par le Front Populaire en 1937 et associé à son pendant légal créé au lendemain de la seconde guerre mondiale, le MTLD, le Mouvement pour le triomphe des libertés démocratiques. Cette généalogie peut être questionnée sous deux

1. Lors de l'élection présidentielle de 1999, les candidats nés avant le mois de juillet 1942 devaient attester de leur participation à la révolution algérienne. Ils devaient par ailleurs être de confession musulmane. 
angles différents : d'abord celui de la continuité réelle entre les différentes organisations ${ }^{1}$ ensuite celui de l'exclusion factice des autres organisations politiques de cette généalogie. L'UDMA, l'Union démocratique du Manifeste algérien, ou le Parti communiste algérien sont les principales victimes de cette occultation qui se fait sous différents prétextes. Certains groupes sont accusés de prôner les réformes sociales avant l'indépendance nationale. D'autres sont écartés pour avoir défendu une conception de la société mixte incluant à la fois les " musulmans " et les "Européens " d'Algérie. L'UDMA, créée par Ferhat 'Abbas en 1946, cristallise les critiques. Accusée d'abord de "réformisme ", elle est également qualifiée de parti bourgeois, le pire des défauts aux yeux d'un parti populiste. Enfin, c'est un parti majoritairement francophone, qui questionne la définition de la nation algérienne, arabe et musulmane. C'est donc à trois obstacles sérieux que se heurte l'intégration de l'UDMA dans la généalogie nationaliste.

Le parti de Ferhat 'Abbas se distingue non seulement du nationalisme populiste du PPA-MTLD mais aussi de ce qu'on a appelé le "réformisme musulman ", incarné principalement par l'association des 'Ulamâ', association qui a eu un devenir bien différent dans l'histoire officielle. En effet, bien qu'étant à la fois un parti réformiste et un parti de notables, cette association a eu un rôle primordial dans la conception arabe et musulmane de la nation. Son créateur, Ibn Badis occupe en conséquence une place particulière dans l'histoire officielle du mouvement national : c'est la seule personnalité du $20^{\mathrm{e}}$ siècle à être commémorée dans des manifestations officielles ou dans les manuels scolaires. En revanche, le parti de Ferhat 'Abbas fait l'objet de stéréotypes qui ont la vie dure. Il est accusé d'une modération qui frise la mollesse ; on lui reproche de rassembler

1. C'est ce que fait Mohammed Harbi, Le FLN mirage et réalité, des origines à la prise du pouvoir (1945-1962), Paris, Les éditions Jeunes Afrique, 1985, 446 p., p. 17. des politiciens bourgeois travaillant en bonne intelligence avec des hommes politiques français, coupés du peuple et perdus dans de vaines stratégies électoralistes. L'histoire officielle écarte donc la question de savoir quel a été en réalité l'apport politique, culturel et militant de l'UDMA au mouvement nationaliste.

\section{○ 'ALI BOUMENDJEL, UN PARCOURS MILITANT}

Choisir une approche biographique pour aborder cet aspect permet de remettre en question l'histoire officielle. Dans l'historiographie officielle algérienne, la biographie n'existe que pour des shuhadâ', des martyrs, et selon les formes figées de l'hagiographie. En dehors de ces formes figées, qui se retrouvent aujourd'hui encore dans la publication de brochures biographiques sur les héros de la nation algérienne, le slogan qui prévaut est celui selon lequel il n'y a eu durant la guerre de libération nationale " qu'un seul héros : le peuple ". Les parcours d'hommes engagés dans les luttes de pouvoir de l'après indépendance doivent s'effacer. Peu de biographies sortent de ces formes rigides et cloisonnées destinées à renforcer une histoire officielle forte et dogmatique que l'étude de parcours individuel pourrait contribuer à ébranler.

Plutôt que de choisir un dirigeant de l'UDMA, tel qu'Ahmed Boumendjel ou Ahmed Francis, précédés par leurs réputations d'hommes politiques et dont les parcours sont déjà connus dans les grandes lignes, on peut suivre l'itinéraire de 'Ali Boumendjel, l'avocat algérois arrêté et assassiné en mars $1957^{2}$. Sans être un mili-

2. Les circonstances de sa mort et leur résurgence au moment de la publication des mémoires du général Aussaresses ont fait récemment resurgir le nom de 'Ali Boumendjel. Officiellement, 'Ali Boumendjel s'est suicidé en mars 1957, alors qu'il était détenu par les parachutistes. Dans ses mémoires, Aussaresses reconnaît qu'il a lui-même donné l'ordre de le précipiter du haut d'un immeuble d'El Biar, ce que beaucoup subodoraient depuis 1957. Paul Aussaresses, Services Spéciaux Algérie 1955-1957, Paris, Perrin, 2001, 197 p., p. 173 et suiv. 
tant moyen, il demeure une figure très discrète de la direction de l'UDMA.

Boumendjel est issu d'une famille originaire des Beni Menguellet. Son père est devenu instituteur après être passé par l'école normale de Bouzareah. Ce métier oblige la famille à quitter la région pour s'installer dans le Sud, puis à Relizane, où 'Ali Boumendjel est né en 1919 et enfin à Larb'a, à proximité d'Alger. La famille évolue dans un milieu d'instituteurs : les soeurs de 'Ali sont devenues institutrices et se sont mariées à des collègues de leur père. Elles sont "émancipées ", selon les termes de l'époque et font partie des quelques femmes de la ville qui sortent sans voile. Ahmed, le frère aîné de 'Ali, né en 1908, a lui aussi "fait la Bouzareah" avant de partir étudier le droit à Paris pour devenir un brillant avocat. Les frères et sours de 'Ali parlent le kabyle à leur mère, le français à leur père et l'arabe dans la rue. Il s'agit d'une famille d'intellectuels comme en témoigne un Sadeq Hadjérès émerveillé par la bibliothèque familiale ${ }^{1}$.

L'engagement politique familial commence à la génération du père, Mohand Boumendjel qui anime dans les années 1930 et 1940 à Larb‘a une association, Nadi el Islah (cercle de la réforme), qui organise à la fois des rencontres culturelles, un groupe de scouts et dispose d'une salle de prière. Au sein du conseil d'administration, il représente la tendance laïque qui s'oppose parfois (et toujours respectueusement) à une tendance religieuse. En 1946, il prend fait et cause pour l'UDMA portant, malgré son âge, la contradiction dans des réunions électorales du MTLD $^{2}$. Le frère de 'Ali, Ahmed, de 11 ans son aîné est installé comme avocat à Alger. Il est d'abord proche du PPA et de Messali dont il est l'avocat. Élu conseiller municipal pour Alger en 1938, il se rapproche de Ferhat 'Abbas et devient son principal lieutenant au sein des AML, l'association

1. Entretien avec Sadeq Hadjérès.

2. Sadeq Hadjérès, Toile de fond des années 1940, Texte inédit. des Amis du Manifeste et de la liberté. L'organisation est créée à la suite de la rédaction d'un texte révolutionnaire et nationaliste. Le Manifeste du peuple algérien, daté du 10 février 1943, condamne en effet la colonisation et revendique le droit du peuple algérien à disposer de soi. Il exige la rédaction d'une constitution garantissant la liberté et l'égalité des habitants sans distinction de "race " et de religion, la reconnaissance de l'arabe comme langue officielle au même titre que le français, la liberté de culte dans le respect de la séparation de l'Église et de l'État. Associé à un Additif exigeant à la fin de la guerre une Assemblée algérienne constituante, ce texte réalise l'exploit remarquable de rassembler à la fois les partisans de Messali Hadj et ceux de Ferhat 'Abbas. Il constitue, selon l'expression de Benjamin Stora, la majorité politique du peuple algérien ${ }^{3}$. Il est rejeté par les autorités françaises, qui proposent des réformes dérisoires dans le contexte des années 1943-1944, et devient la plate forme revendicative de référence pour l'ensemble du mouvement national dans l'après-guerre. Les AML sont créés en mars 1944 pour le défendre.

Cette union disparate se maintient jusqu'aux événements de mai 1945. L'implication du PPA dans les manifestations puis la terrible répression du Constantinois ont raison de la précaire entente. Les partisans de Messali retrouvent leur ancienne organisation, le PPA. Ferhat 'Abbas rassemble progressivement les siens : lors les élections législatives de juin 1946, il lance une nouvelle organisation, l'UDMA, l'Union démocratique du Manifeste algérien.

La forme particulière de l'engagement de 'Ali Boumendjel au sein de l'UDMA permet d'éclairer les pratiques politiques en vigueur à l'intérieur de son parti, son fonctionnement et le contenu idéologique véhiculé par les militants et les élus. Son

3. Benjamin Stora et Zakya Daoud, Ferbat Abbas une utopie algérienne, Paris, Denoël, coll. "L'aventure coloniale de la France ", 1995, 429 p., p. 121. 
étude relance la discussion sur leur rapport avec l'histoire officielle. Ainsi, le parcours de 'Ali Boumendjel révèle que, contrairement au stéréotype, il existe à l'intérieur de l'UDMA une véritable radicalité politique portée par un courant militant dynamique. Par ailleurs, le parti propose une définition de la nation algérienne et contribue à l'écriture d'une histoire nationale. Évidemment cette histoire nationale et cette conception de la nation s'écartent de la nation entendue au sens du PPA et des 'Ulamâ'. Reste à éclairer comment s'effectue dans l'UDMA le passage de l'assimilationnisme à un projet d'Algérie indépendante. Quelle est, d'autre part, la définition de la nation produite par l'UDMA ? Enfin, comment les militants UDMA se sont-ils ralliés au FLN?

\section{O DE L'ASSIMILATION À L'INDÉPENDANCE}

Avant la seconde guerre mondiale, Ferhat 'Abbas appartenait à un courant politique héritier du mouvement Jeune Algérien. Il était alors proche du Docteur Bendjelloul et incarnait le courant assimilationniste. Influencé par la Nahda, le mouvement de renaissance arabe, en même temps que par les références européennes, il a défendu la possibilité d'un compromis, d'une synthèse permettant aux colonisés d'exister en France avec leur histoire et leur culture. Cette assimilation s'appuyait sur la croyance dans la "vraie France", démocratique et généreuse qui devait intégrer progressivement ses enfants musulmans.

Avec le Manifeste, 'Abbas et ses partisans basculent dans l'indépendantisme. Pourtant, l'accusation d'assimilationnisme s'est perpétuée jusqu'à aujourd'hui. " L'Algérie en tant que patrie est un mythe. [...] j'ai visité les cimetières : personne ne m'en a parlé ${ }^{1}$. "Que de fois cette célèbre phrase de Ferhat 'Abbas a-t-elle été citée contre lui ! Au point que 'Ali Boumendjel la re-

1. B. Stora et Z. Daoud, Ferhat Abbas..., op. cit., p. 73. prend pour valoriser au contraire l'évolution intellectuelle de "Abbas : "On reproche à Abbas d'avoir changé. On lui rappelle sa fameuse phrase ; "J’ai cherché partout la nation algérienne... et je ne l'ai pas trouvée." C'est qu'alors, elle n'existait pas, en effet. Et il le disait. Honnêtement. Aujourd'hui elle existe. Il la trouve. Et il le dit. Honnêtement, toujours. La nation algérienne en est au stade où l'être vient de sortir du néant pour entrer dans la vie. C'est un organisme en formation et dont le développement est particulièrement rapide $^{2}$."

D’une génération plus jeune que Ferhat 'Abbas et même que son frère Ahmed, 'Ali Boumendjel entre en politique dans les années 1942-1943 avec le Manifeste, sur la base d'une revendication d'indépendance. Dès 1943, il écrit de très nombreux articles dans le journal des AML, Égalité, qui devient par la suite la République Algérienne, organe de l'UDMA. La plupart de ses textes a pour objet une critique dure de la politique française en Algérie. Il manie pour ce faire une langue française très soutenue, alliant des références littéraires recherchées et une mordante ironie. Ses lecteurs ne se comptent pas seulement dans les rangs de l'UDMA : ses camarades ou enseignants de la faculté de droit comme AlbertPaul Lentin ou René Capitant, ses amis du PCA, Henri Alleg ou Sadeq Hadjérès le lisent. Ils apprécient particulièrement les chroniques humoristiques signées Ali Beaurond dans lesquelles il mène une charge en règle contre les intérêts économiques gouvernant la politique coloniale de la France. La politique d'assimilation est régulièrement l'objet de son humour ravageur. Il écrit ainsi en 1946 :

2. Égalité, n 49, novembre 1946, article signé Juba III. Il faut remarquer le caractère presque blasphématoire au regard de l'histoire officielle de l'historicité affirmée de la nation algérienne, d'une nation algérienne née dans un passé récent. Pour le PPA, la nation algérienne préexiste à la colonisation. Se dessinent déjà deux histoires de la nation et du mouvement national. 
"En l'an de grâce 1946, l'indigène d'Algérie estime, en effet, que "l'encombrante" politique d'assimilation est une conception désuète, qui se rattache dans l'histoire de l'Algérie, à une époque révolue, celle du complexe d'infériorité. Pendant longtemps, l'Arabe ou le Kabyle, à force d'être traité par l'Européen en inférieur, avait fini par se juger tel. Honteux de lui-même et de ses compatriotes, il allait, rasant les murs et courbant l'échine, quelle que soit sa distinction, sa richesse ou sa culture personnelle. Tandis que la situation diminuée de la masse était sans issue, on offrait à une "élite" infinitésimale une planche de salut : "l'assimilation". On lui demandait de renoncer à son milieu originel, de s'en détacher, de s'en désolidariser, pour se fondre dans un milieu nouveau. Il s'agissait, autant que faire se peut, de perdre l'accent du terroir, de boire du vin, d'apprendre à faire correctement un noud de cravate et à penser français ${ }^{1}$. "

La violence du ton et l'amertume des critiques ne sont pas le fait du seul 'Ali Boumendjel. On les retrouve également dans les éditoriaux de Ferhat 'Abbas ou chez d'autres collaborateurs du journal, Ahmed Boumendjel, Ahmed Francis, Kaddour Sator, Kadda, Boutarène... Elles provoquent des réactions d'enthousiasme ou de rejet : la censure s'abat sur le journal à plusieurs reprises, à tel point que Francis doit s'expliquer devant le bureau politique d'avoir adouci certains textes afin d'éviter les lourdes amendes mettant en péril le budget de la République Algérienne. Les lecteurs s'accommodent donc d'espaces blancs qui entrecoupent les articles. Le style des textes de 'Ali Boumendjel rappelle les interventions des élus de l'UDMA à la seconde Assemblée nationale constituante et révèle un paradoxe apparent. À la tribune de l'Assemblée comme dans la presse, les udmistes suscitent des réactions beaucoup plus violentes que le PPAMTLD.

En juin 1946, en effet, l'UDMA connaît un succès électoral important puisque onze élus de ce parti, dont 'Abbas lui-

1. Égalité, n 49, novembre 1946. même, représentent le $2^{\mathrm{e}}$ collège ${ }^{2}$. S'engageant pleinement dans le travail parlementaire, ils présentent un texte de loi portant création d'une République algérienne unie à la France par un lien fédéral. Au cours de leurs discours, les élus insistent, pour amadouer l'auditoire, sur les importantes concessions faites dans ce texte et sur le lien entre les deux pays. Dans les réunions organisées en Algérie au contraire, les discours insistent davantage sur la rupture. Y voir une ambiguité ou une hypocrisie électorale serait simpliste : pour ces élèves de l'école française, l'indépendance n'est pas synonyme de rupture violente mais bien plutôt d'une évolution historique nécessaire pour faire advenir les valeurs communes de la démocratie. Pourtant, toutes les interventions des élus de l'UDMA provoquent une forte tension et des incidents violents. Le contraste est saisissant entre les attentes des élus du $2^{\mathrm{e}}$ collège qui pensent pouvoir s'exprimer enfin au sein de la démocratie française et un auditoire choqué d'entendre pour la première fois des "indigènes " exprimer une opinion critique. Ces hommes incarnent l'assimilation, sont les "produits de l'école française ", des "évolués ", selon les termes d'époque. Ils ne doivent pas sortir de leur position de colonisés, ni contredire les stéréotypes paternalistes coloniaux. Alors que l'état d'esprit des udmistes est résumé dans cette phrase de Ferhat 'Abbas, "cela fait 116 ans que nous attendons ce moment ", l'auditoire l'invective dès qu'il commence à s'exprimer, le qualifiant d'anti-français. Qu'attend-on en effet de ces élus ? Une certaine complaisance et, surtout, de la gratitude. Certains élus du $2^{\mathrm{e}}$ collège ont l'heur de ne pas choquer, ils séduisent par leur "truculence " et leur "pittoresque". Les élus de l'UDMA, eux, n'incarnent aucun exotisme et refusent de se laisser inférioriser dans des relations empreintes d'orientalisme et de paternalisme colonial.

2. Le $2^{\text {e }}$ collège électoral regroupe la population colonisée. En 1946, il est représenté au Parlement français par le même nombre d'élus que le $1^{\text {er }}$ collège. 
Ils jouent leur rôle d'élus avec sérieux dans le but de promouvoir de réelles réformes et c'est leur présence même à la tribune qui paraît contestée.

Quant aux élus du MTLD, leurs interventions sont bien plus brèves : elles ont pour objet de nier à l'Assemblée toute légitimité pour légiférer sur l'Algérie. Ils exigent une Assemblée algérienne constituante pour déterminer le nouveau statut de l'Algérie et pourtant, leurs interventions ne suscitent pas de réactions aussi vives. C'est donc bien la personnalité des élus UDMA, leur manière de porter la critique dans les formes (pratiques, vêtement, sociabilité, langage) de la vie politique démocratique et cette intrusion de "l'indigène " dans un champ réservé où il prend son autonomie qui sont intolérables. De la même manière, la dureté du ton des articles de Boumendjel dans la République Algérienne vient autant du fond que de cette forme caractéristique.

Cette radicalité, associée à un projet défendant clairement l'indépendantisme pose question : pourquoi en effet les militants de l'UDMA ne sont-ils jamais qualifiés de nationalistes, au même titre que les PPA-MTLD ? L'UDMA propose une définition de la nation et de la République algérienne qui diffère radicalement de celle du PPA. C'est probablement dans cette différence qu'il faut rechercher des éléments de réponse.

\section{○ QUELLE NATION ALGÉRIENNE ?}

Les articles de 'Ali Boumendjel contribuent à la définition de la société de la future République algérienne. Contrairement à la définition qu'en donne le PPA ou les 'Ulama' fondée sur la langue arabe et l'islam, la société algérienne envisagée par l'UDMA englobe la population de l'Algérie dans toutes ses minorités. Les juifs et les "Européens " d'Algérie sont explicitement cités comme faisant partie de la société algérienne. À plusieurs reprises, les diri- geants expriment, au cours de meetings, le désir de voir des "Européens " adhérer à l'UDMA, comme l'a fait Roland Miette, élu de l'UDMA, qui écrit dans la République algérienne. 'Ali Boumendjel ne limite d'ailleurs pas son activité militante aux réunions de l'UDMA. Il multiplie les occasions de rencontres avec des militants d'autres tendances : il fréquente les locaux d'Alger Républicain, le journal communiste, un lieu de rencontre prisé par les militants " européens " et " musulmans ", par des artistes ou encore des sportifs. Il compte parmi ses proches des militants du PCA, mais aussi des membres d'associations chrétiennes, des intellectuels avec lesquels il se trouve plus d'affinités sans doute qu'avec certains militants du PPA. La question kabyle, elle, n'apparaît pas en tant que telle dans la République algérienne: les habitants "indigènes " de l'Algérie sont appelés indifféremment "arabes et berbères " ou désignés simplement sous le terme générique "arabes " dans les textes de 'Ali Boumendjel ${ }^{1}$. Cette conception qui considère l'intégration des minorités et des tendances intellectuelles et politiques comme un enrichissement s'oppose à celle du PPA-MTLD, puis celle du FLN, selon laquelle les minorités doivent au contraire disparaître, leur existence risquant de remettre en question l'unité de la nation.

Il ne s'agit cependant pas de dire que langue arabe et religion musulmane n'ont pas un rôle particulier. Bien au contraire, l'on trouve même dans les articles des frères Boumendjel (pourtant très laïcs) une volonté farouche de défendre l'islam contre les tentatives de l'Administration française de le contrôler. La religion, comme la langue, sont des éléments essentiels de l'identité algérienne, mais elles demeurent des objets d'ouverture et de dialogue, en aucun cas une fermeture et une exclusion de la nation, comme c'est le cas

1. Il dénonce en revanche la politique française visant à diviser la population algérienne, évoquant par exemple la " discrimination criminelle du Berbère et de l'Oriental ". La République algérienne, n² 289, 25 janvier 1952. 
dans le nationalisme du PPA. De nombreux articles de la République algérienne ont une portée historique et culturelle : ils constituent en somme les éléments épars d'une histoire nationale en construction. L'on y trouve par exemple des articles sur l'histoire de l'Algérie, de l'islam et des Arabes ou sur l'histoire de la littérature arabe, de la religion, de la médecine.

\section{O UNE PRATIQUE POLITIQUE DIFFÉRENTE}

Cette conception différente de la nation et de l'histoire nationale est complétée par une pratique politique différente de celle du PPA-MTLD, pratique qui participe du projet de République algérienne défendu par le parti. L'importance accordée dans les réunions de l'UDMA à des stratégies et des négociations électorales pourrait confirmer le stéréotype d'un parti engagé dans des batailles électorales sans fin. Cependant, l'engagement politique d'un 'Ali Boumendjel conduit à relativiser l'importance de cet électoralisme dans la pratique militante de l'UDMA. Alors qu'il prend progressivement des responsabilités dans l'appareil, au sein de la section "Alger-centre " puis dans la Fédération d'Alger et enfin, à partir de 1954, dans le Comité Central et le Bureau Politique, 'Ali Boumendjel ne s'engage en effet jamais activement dans le jeu électoral. Son ami et collègue 'Amar Bentoumi, militant au PPA, s'amuse du fait qu'il est généralement le candidat des causes perdues, au point de le surnommer en riant kebch ed-dabia, le mouton du sacrifice. Il se présente pour les élections où l'UDMA n'a aucune chance, soit en raison des trucages administratifs soit du fait de la concurrence d'autres partis. Ainsi, en 1951, il se présente aux élections à l'Assemblée Algérienne dans la circonscription de Blida, puis en 1954 dans la circonscription de Maison-Carrée ${ }^{1}$. Pourtant, il ne semble

1. À Maison-Carrée par exemple, malgré la très forte implantation du PCA, c'est un candidat "indépendant " qui est élu. Pour les militants, "indépendant " est bien souvent le synonyme "d'administratif". pas y accorder une grande importance. Ces circonscriptions sont perdues d'avance, il faut seulement faire acte de présence et discuter inlassablement, rencontrer les militants, échanger avec les autres forces politiques dans un but de construction politique et d'union.

Outre les rencontres à Alger Républicain, il côtoie André Mandouze au sein du Mouvement mondial pour la paix et participe avec lui à la première conférence de ce mouvement organisée en 1949, salle Pleyel, à Paris. Par la suite, il assiste à une ou deux conférences chaque année et se rend pour cela notamment à Helsinki, Prague ou Paris. Ces réunions, qui lui donnent l'occasion de rencontrer des représentants de différents pays, correspondent bien à sa conviction que l'Algérie participe à un processus mondial de libération des peuples dominés. Par ailleurs, ses amis lui demandent fréquemment de donner des conférences sur l'histoire ou les questions géopolitiques : il évoque l'histoire récente de la Tunisie, la création de la $\mathrm{CED}^{2}$. À travers ses conférences et ses articles, il situe l'Algérie dans le contexte d'une histoire non seulement arabe et musulmane mais aussi mondiale. 'Ali Boumendjel joue donc le rôle d'un polémiste, d'un enseignant et au final, d'un intellectuel cherchant à analyser, à accompagner et à encourager cette révolution que le monde est en train d'accomplir. L'importance accordée à l'enseignement ne lui est pas propre : scolarisation, formation, enseignement participent de la pratique politique de l'UDMA et sont considérés comme essentiels à la constitution en cours de la nation algérienne.

\section{O LE RALLIEMENT AU FLN}

Au sein même de l'UDMA, 'Ali Boumendjel participe à un mouvement contestataire. À partir de 1951, en effet, l'UDMA

2. Il est invité par la JUDMA, la jeunesse de l'UDMA. Mme Boumendjel se souvient également qu'il fait des conférences devant un groupe de séminaristes. Entretien avec Malika Boumendjel. 
entre dans une période de crise politique et financière, qui culmine en 1954. Plusieurs phénomènes sont concomitants : les difficultés économiques au sein de l'UDMA ; l'influence croissante d'Ahmed Boumendjel dans le parti, malgré son installation à Paris en 1950-1951 ; l'apparition d'un courant "jeune-UDMA " dont 'Ali Boumendjel est une des voix ${ }^{1}$. De nombreux dirigeants des sections locales, comme les militants de la JUDMA, la Jeunesse de l'UDMA, se plaignent de ne pas toujours comprendre les stratégies politiques de leurs leaders. Le terme qu'ils utilisent le plus souvent dans leurs critiques est celui d'immobilisme. Les critiques s'intensifient après le $1^{\text {er }}$ novembre 1954 : dès lors, la stratégie des leaders apparaît encore moins compréhensible et l'immobilisme devient insupportable. En effet, les tentatives de rapprochement avec le FLN sont très discrètes et invisibles aux yeux des militants. En août 1955, une délégation de la JUDMA est reçue par Ahmed Boumendjel. Les délégués reprochent à la direction son inactivité et expriment le désir de participer à la lutte pour l'émancipation du peuple algérien. D'autre part, ils souhaitent être éclairés sur la stratégie du parti. Ahmed Boumendjel leur répond, vertement à son habitude, que l'UDMA est fidèle à ses principes et que "si l'on veut aller se faire tuer dans les Aurès ", il n'y voit aucun inconvénient ${ }^{2}$.

De nombreux documents font état d'un "vent de désagrégation" qui souffle sur

1. Une partie de la tendance "jeune-UDMA " semble souhaiter la prise en main du parti par Ahmed Boumendjel, jugé plus radical et dynamique que Ferhat 'Abbas. Il n'est pas certain qu'Ahmed Boumendjel ait souhaité en profiter pour prendre le contrôle du parti. Rapport du préfet de Constantine, janvier-février 1953, ministère des Affaires algériennes, CAOM, 81F 771.

2. Note de renseignement PRG, Alger, 30 août 1955, CAOM, 1K 833*. La plupart des militants ignore sans doute les activités clandestines d'Ahmed Boumendjel qui font dire aux informateurs de la police, à l'occasion d'une rencontre avec le sultan du Maroc, en novembre 1955: "Cette démarche de $\mathrm{M}^{\mathrm{e}}$ Ahmed Boumendjel s'inscrit dans la ligne nouvelle de l'UDMA qui, jusqu'ici cantonnée dans l'action politique pure, se range désormais aux côtés des combattants du FLN et de l'Armée de Libération. " Note de renseignement, PRG, Alger, 8 novembre 1955, CAOM, 4I122/16**
l'UDMA depuis le début des années 1950. Lors du congrès du parti au mois de septembre 1951, à Constantine, il est question de prendre des décisions de fond. Ahmed Boumendjel doit affirmer publiquement que ce congrès ne sera pas celui de la "liquidation" du parti, mais bien plutôt de sa réforme, trahissant ainsi la profondeur de l'inquiétude des militants ${ }^{3}$. En juillet 1952, Ferhat 'Abbas est vaincu alors qu'il se présente à l'élection pour le remplacement du député Kessous, décédé. La rumeur veut qu'il envisage un retrait de la politique ${ }^{4}$.

L'une des sections les plus contestataires est celle d'Alger-centre. Ses dirigeants adressent leurs questions au Comité Central, menaçant de démissionner si les réponses données ne sont pas satisfaisantes. Les militants de la section s'interrogent sur les tentatives d'union des partis nationalistes au sein d'un Front algérien ${ }^{5}$ ou critiquent en août 1953 le manque de réaction des dirigeants à la suite de la déposition du sultan du Maroc. L'administration remarque, dans le même temps, que de nombreux militants rejoignent les rangs du PPA. En janvier 1953, plusieurs sections renvoient les cartes annuelles du parti, protestant contre les derniers mots de la carte : " Notre raison d'être : libérer et s'unir. Une Algérie libre au sein d'une Afrique du Nord fédérée, en coopération avec une France libérale et démocratique ${ }^{6}$. "

À partir de l'année 1954, 'Ali Boumendjel participe aux activités de la section d'Alger-centre. Il est nommé secrétaire général de la section en mars 1954 et se charge de la liaison avec le comité central ${ }^{7}$.

3. Document de police du 15 septembre 1951, ministère des Affaires algériennes, CAOM, 81F771.

4. Synthèse mensuelle de police, septembre 1952, ministère des Affaires algériennes, CAOM, 81F771 et rapport du préfet de Constantine, janvier-février 1953, ministère des Affaires algériennes, CAOM, 81F711.

5. Synthèse de police du 23 septembre 1952, ministère des Affaires algériennes, CAOM, 81F711.

6. Document de police du 26 janvier 1953, ministère des Affaires algériennes, CAOM, 81F771.

7. Document de police du 9 mars 1954, ministère des Affaires algériennes, CAOM, 81F771. 
Le même mois, au cours d'une réunion de la section, il prononce un véritable réquisitoire. C'est l'absence de communication entre les sections locales et le comité central qui, selon lui, pose problème. Ses attaques contre l'organisation se multiplient. Mais, s'il se montre très critique visà-vis du fonctionnement du parti et de la politique menée par la direction de l'UDMA, il ne veut en aucun cas que Ferhat 'Abbas soit écarté. Bien au contraire, il propose son retour aux affaires comme solution au profond malaise du parti ${ }^{1}$. D'ailleurs, dit-il, ses critiques ont abouti à des résultats tels que l'organisation d'une conférence des cadres et une virulente lettre de Ferhat 'Abbas aux membres du comité central leur reprochant leur inaction durant l'année écoulée : de quoi redynamiser le parti.

À la conférence des cadres, en avril 1954, 'Ali Boumendjel participe à la commission d'organisation qui propose des orientations nouvelles. Parmi les réformes proposées, la multiplication des réunions du secrétaire général et du comité central ainsi que l'établissement par avance d'une nouvelle conférence des cadres en septembre pour évaluer l'efficacité de la mise en ouvre des réformes ${ }^{2}$. Cependant, devant l'absence de réformes concrètes, 'Ali Boumendjel démissionne au mois de mai de ses mandats de secrétaire général de la section d'Alger-centre et de responsable de la région algéroise. Il affirme qu'"aucun effort n'a été fait pour donner une nouvelle impulsion à l'UDMA ${ }^{3}$ ".

Ainsi, en 1954, 'Ali Boumendjel est donc devenu une des personnalités montantes du parti, et un animateur de la tendance jeune-UDMA. S'il propose des changements dans l'organisation du parti, exige

1. Document de police du 23 mars 1954, ministère des Affaires algériennes, CAOM, 81F771.

2. Note de renseignement de police non datée, ministère des Affaires algériennes, CAOM, 81F771.

3. Document de police du 14 mai 1954, ministère des Affaires algériennes, CAOM, 81F771. une politique plus active et intransigeante et menace lorsque les réformes ne viennent pas, le déroulement de la révolution le conduit à développer également une activité souterraine.

$\mathrm{Au}$ lendemain du soulèvement du $1^{\mathrm{er}}$ novembre 1954, 'Ali Boumendjel est très inquiet devant la tournure que peuvent prendre les événements ${ }^{4}$. Un élément intervient qui facilite son ralliement au FLN : l'accession d'Abbane Ramdane à la tête de la région d'Alger pour le FLN, à la suite de sa libération le 19 janvier 1955. Dès son arrivée, Abbane recherche tous les contacts possibles. Il a pour objectif de rallier au FLN les forces politiques dynamiques et s'intéresse donc naturellement à la position du PCA et de l'UDMA. Comme 'Ali Boumendjel, Abbane a fait ses études au collège de Blida, une pépinière de nationalistes. Abbane et Boumendjel se connaissent et la présence du premier à la tête du FLN a, pour le second, un caractère rassurant. Il ne s'agit pas d'un aventurier mais d'un homme politique, entouré d'autres intellectuels amis de Boumendjel comme Benyoucef Benkhedda et Sa'ad Dahlab, avec lesquels il est prêt à travailler ${ }^{5}$.

Alors que "Abbane cherchait tous azimuts ce qui pouvait donner une consistance politique à l'insurrection ", selon l'expression de Hadjérès, son ancien camarade de classe Boumendjel devient alors son interlocuteur privilégié dans l'UDMA. Les contacts entre les deux hommes permettent à Abbane d'organiser une action spectaculaire : encourager les élus, notamment les élus "préfabriqués " par l'Administration, à prendre position ${ }^{6}$. En effet Bendjelloul lui-même, incarnation

4. Entretien avec Me Mohammed Hadj Hamou.

5. Benkhedda et Dahlab sont également d'anciens camarades du collège de Blida.

6. "Abbane Ramdane est tenu au courant de l'évolution des esprits grâce aux contacts qu'il entretenait avec 'Ali Boumendjel "rapporte ainsi Henri Douzon, l'avocat et ami d'Abbane Ramdane. Henri Douzon, "Les occasions perdues ", in Henri Alleg et Henri Douzon (dir.), La Guerre d'Algérie, t. 1, L'Algérie des origines à l'insurrection, Paris, temps Actuels, p. 568-569. 
de la politique d'assimilation, prend alors ses distances, et met en avant une revendication nationale désormais, dit-il, incontournable. L'action des militants de l'UDMA comme 'Ali Boumendjel, Ahmed Francis et Kaddour Sator, aboutit finalement à la publication, le 26 septembre 1955, de la "déclaration des 61 ":

" [E]n raison de la gravité des événements qui traversent l'Algérie [...] analysant les raisons profondes des troubles actuels, [les élus] affirment solennellement qu'elles sont essentiellement d'ordre politique. Ils sont ainsi conduits à constater que la politique "d'intégration" [...] est actuellement dépassée. L'immense majorité des populations est présentement acquise à l'idée nationale algérienne. Interprètes fidèles de cette volonté, les élus soussignés croient de leur devoir d'orienter leur action vers la réalisation de cette aspiration ${ }^{1}$."

Ce rôle d'intermédiaire entre Abbane et l'UDMA conduit par ailleurs 'Ali Boumendjel à maintenir le contact entre son frère Ahmed, alors avocat à Paris, et le FLN. À plusieurs reprises, Ahmed fait le déplacement à Alger. 'Ali organise et participe à des réunions clandestines, sans que l'on puisse déterminer avec exactitude qui assistait à ces rencontres. Le FLN a ainsi très tôt courtisé l'UDMA. Des contacts sont attestés entre le FLN et l'UDMA à partir d'avril 1955. Pour un Abbane, dirigeant du FLN doté d'une réelle conception politique de la construction du Front, les partisans de Ferhat 'Abbas doivent contribuer à l'élaboration du projet politique de la révolution et à la lutte pour l'indépendance. Kiouane a alors fourni un projet d'union entre les deux partis et 'Ali Boumendjel participe, en mai 1955, à une commission chargée d'étudier le projet pour le bureau politique de l'UDMA. Les instances du parti choisissent finalement de rejeter cette pro-

1. Henri Douzon, "Les occasions perdues", op. cit., p. 569. position ${ }^{2}$. Ce n'est qu'avec le ralliement de Ferhat 'Abbas lui-même, en avril 1956, que l'UDMA se dissout dans le FLN.

Pourquoi un homme aussi radical et dynamique que 'Ali Boumendjel n'a-t-il pas quitté l'UDMA pour le PPA ? La tradition familiale a peut-être joué un rôle, l'engagement d'un frère aîné dans la direction du parti conduisant le cadet à une forme de loyauté familiale. Mais il y a également de réelles raisons politiques à ce choix : 'Ali Boumendjel rejette le messalisme en vigueur au sein du PPA-MTLD, son organisation empruntée à la clandestinité et son conservatisme $^{3}$. Il cherche au contraire à réintroduire de la nuance et de la complexité dans l'étude de l'histoire, des mouvements politiques et des relations internationales. Cette conscience inquiète ne peut être à sa place dans un parti défendant un programme populiste et fonctionnant sans souci démocratique. Malgré l'immobilisme et parfois le manque de radicalité des positions des dirigeants, l'activité et le projet politique et social de l'UDMA lui conviennent davantage.

Le ralliement des partisans du Manifeste au FLN a contribué à modifier le regard porté sur les militants nationalistes : à la mort de 'Ali Boumendjel, nombre de ses amis et collègues témoignent dans certains journaux (Le Monde, France Observateur, L'Express, Libération, Témoignage Chrétien...) de la justesse et de la légitimité de ses prises de positions par le passé. Dès lors, les partisans du FLN n'apparaissent plus seulement comme des "terroristes" ou des "fellaghas ". Des hommes modérés, "évolués ", militants des causes justes prennent partie pour l'organisation. La cause s'en trouve légitimée dans les milieux dont l'UDMA était le plus proche et des intellectuels, des militants de la paix, des chrétiens engagés participeront au

2. Cette étrange affaire n'est pas confirmée par ailleurs. Nous ne disposons que de quelques renseignements de police, notamment une note de renseignement des PRG du 4 mai 1955, CAOM, 1K 833*.

3. Mohammed Harbi, Le FLN..., op. cit., p. 26. 
mouvement de contestation qui se développe en France durant l'année $1957^{1}$.

Le ralliement au FLN de 'Ali Boumendjel et des militants de l'UDMA est apparu lié à l'existence d'un courant politique au sein du FLN incarné par Abbane Ramdane et ses amis centralistes. L'élimination d'Abbane fin 1957 a posé la question de l'interruption de cette dynamique constructive et du rôle des anciens udmistes dans le FLN.

1. P. Vidal-Naquet rappelle qu'après le " grand silence de 1956, à nouveau en 1957, la protestation s'est élevée ". Elle débute à la suite de l'affaire Boumendjel et se prolonge dans les affaires Bouhired, Alleg et Audin. Pierre Vidal-Naquet, Face à la raison d'État un historien dans la guerre d'Algérien, Paris, La Découverte, 1989, 259 p., p. 31.
Mais, au-delà, l'occultation de l'UDMA dans l'histoire officielle n'est pas sans rapport avec cet assassinat et l'élimination d'un courant politique au sein du FLN ${ }^{2}$.

2. Gilbert Meynier, Histoire intérieure du FLN 1954-1962, Paris, Fayard, 2002, 812 p., p. 698.

Malika Rahal est agrégée d'histoire, professeur dans le secondaire en Seine-Saint-Denis. Elle mène actuellement une thèse sur l'UDMA sous la direction de Benjamin Stora à l'INALCO. Son DEA s'intitulait : "'Ali Boumendjel (1919-1957), itinéraire d'un bomme de bonne volonté". 